\title{
Experience of monitoring the recurrent laryngeal nerve in thyroid surgery with endotracheal intubation
}

\author{
Feng Liang ${ }^{1}$, Guifeng $\mathrm{Liu}^{2}$, Huiying $\mathrm{Li}^{1}$, Yunpengfei $\mathrm{Li}^{1}$ and Xuefeng $\mathrm{Li}^{1, \mathrm{a}}$ \\ ${ }^{1}$ Department of Anesthesiology, China-Japan Union Hospital, Ji Lin University, Changchun, Jilin, China \\ ${ }^{2}$ Department of Radiology, China-Japan Union Hospital, Ji Lin University, Changchun, Jilin, China
}

\begin{abstract}
Purpose:To analysis clinical experience of applying recurrent laryngeal monitoring endotracheal tube (NIM EMG Endotracheal Tube) in the surgery of thyroid.Method:A retrospective analysis of 84 patients who underwent endotracheal intubation laryngeal nerve monitoring by thyroid surgery in the Chinese-Japanese Friendship Hospital of Jilin University from March to December in 2015.To summarize the experience of intubation with NIM EMG Endotracheal Tube.Result 77 (91.7\%)had initial intubation achievement in the 84 patients.FROM the 77 cases we had gotten s atisfactory nerve monitoring signal. Whereas there are 7 cases $(8.3 \%)$ appear abnormal EMG or signal missing,in the 7 cases there is one which being intubated too deep ,3 cases which being intubated too shallow and 3 cases with malrotation intubation.Conclusion:We got the satisfactory signals after adjust ${ }^{1}$ ing the tube by using the visual laryngoscope.
\end{abstract}

\section{Instruction}

Lately laryngeal nerve monitoring (EDM) technology can locate and discriminate the recurrent laryngeal nerve, which can avoid intraoperative recurrent laryngeal nerve injury [1].Recently in surgery of Thyroid intraoperative laryngeal nerve monitoring technology regards the endotracheal tube as a surface electrode EMG which can record electrodes, recording electrodes in contact with the vocal cords is good is directly related to neurophysiological monitoring effects. Whether electrode is in contact with vocal cord well relates to the effect of neurophysiological monitoring directly.Therefore, accurate intubation is an important part of the completion of the recurrent laryngeal nerve monitoring.In this paper, we analyze 84 cases that were in our hospital from March to December in 2015 with various types of thyroid surgery and summarize the experience of intubation.During the surgery we all use the recurrent laryngeal nerve monitoring endotracheal tube. Details as below

\section{Material an method}

\subsection{General information}

84 cases in our hospital for treatment of elective thyroid surgery from March to December in 2015 , their age duration is $22-65$ years old, weighing duration is $48-79 \mathrm{~kg}$, there are 13 male and 71 female

${ }^{\mathrm{a} C}$ Corresponding author: 28186282@qq.com 
cases. Their ASA grades are I- II level. In the 84 cases there are 53 diagnosed as papillary thyroid carcinoma.30 cases as nodular goiter, and one as lymphocytic thyroiditis.

\subsection{Method}

Anesthetizing 30MINS Before being anesthetized patients are given penehyclidine $1 \mathrm{mg}$ by intramuscular injection. After entering the operating room we take the routine testing for them as BP, HR, ECG, SpO2, open upper extremity venous access, give Ringer's solution by intravenous infusion (infusion rate $6-8 \mathrm{ml} / \mathrm{kg} \cdot \mathrm{h}$ ), after the induction of anesthetizing,we start to monitor the concentration of $\operatorname{co} 2$ in the end-tidal of exhaling and the concentration of sevoflurane in inhaled and exhaled process. Induction of anesthesia: intravenous inject midazolam followed by $0.05 \mathrm{mg} / \mathrm{kg}$, acid cis atracurium $0.05 \mathrm{mg} / \mathrm{kg}$, sufentanil $0.5 \mathrm{ug} / \mathrm{kg}$, propofol $2 \mathrm{mg} / \mathrm{kg}$. Ventilation mode: Chose intermittent positive pressure ventilation, make sure the respiratory rate is $12 / \mathrm{min}$, tidal volume $8-10 \mathrm{ml} / \mathrm{kg}$, inspiratory to expiratory ratio is $1: 1.5$, PetCO2 maintained at $30-40 \mathrm{mmHg}$.Intraoperative continuous sevoflurane nitrous oxide - oxygen anesthesia was maintained state of the patient, to maintain the depth of anesthesia in 1.2-1.5MAC.

Before endotracheal intubation we check the package whether there are situation like damage, leaks cuff and form plastic pipe as the desired angle.After induction of anesthesia we stop giving nitrogen to give oxygen.After three minutes, we put the visual laryngoscopes in by left hand and fully reveal the glottis, Then put the NIM EMG endotracheal tube (Medtronic Xomed, USA) into the glottis by right hand, assistant to extubation guide core at this time.Continue send the tube to the subglottic, place the blue electrodes on the exposed parts of the glottis. Slow adjust the tube, ensure the tube electrode is in contact with both sides of the vocal cords fully.After intubation completing, inflate the cuff,make the tube connect to the anesthesia machine then control ventilation, so we can see the PetCO2 waveform and rise and fall movement on both sides of chest. Place the Dental pad and fix the tube to the right side of the mouth. Endotracheal intubation and anesthetizing are completed by the same anesthesiologist operation.

\section{Result}

$77(91.7 \%)$ had initial intubation achievement in the 84 patients.FROM the 77 cases we had gotten $\mathrm{s}$ atisfactory nerve monitoring signal. Whereas there are 7 cases $(8.3 \%)$ appear abnormal EMG or signal missing, in the 7 cases there is one which being intubated too deep ,3 cases which being intubated too shallow and 3 cases with malrotation intubation. We got the satisfactory signals after adjusting the tube by using the visual laryngoscope.

\section{Discuss}

During Thyroid Surgery taking advantage of the technology of recurrent laryngeal nerve monitoring (IONM) can accurately locate and identify the recurrent laryngeal nerve, reduce the risk of recurrent laryngeal nerve injury, reduce postoperative complications. CURRENTLY Medtronic Xomed NIM-Response 3.0 EMG nerve monitor and NIM EMG endotracheal tube are the most advanced monitoring equipment. NIM EMG endotracheal tube used in this study is Medtronic Xomed NIM standard enhanced TM endotracheal tube (diameter of the catheter for men $7.0 \mathrm{~mm}$, women diameter of the catheter $6.0 \mathrm{~mm}$ ). The TUBE has two pairs of recording electrodes implanted in the side wall.IN the front of electrode there is one exposed portion which is about $3 \mathrm{~cm}$ and its color is blue, only the exposed portion of the electrode is located in the middle and the recording electrode is located full access to the vocal cords (Fig. 1), we ensure receiving the most accurate EMG signal. 


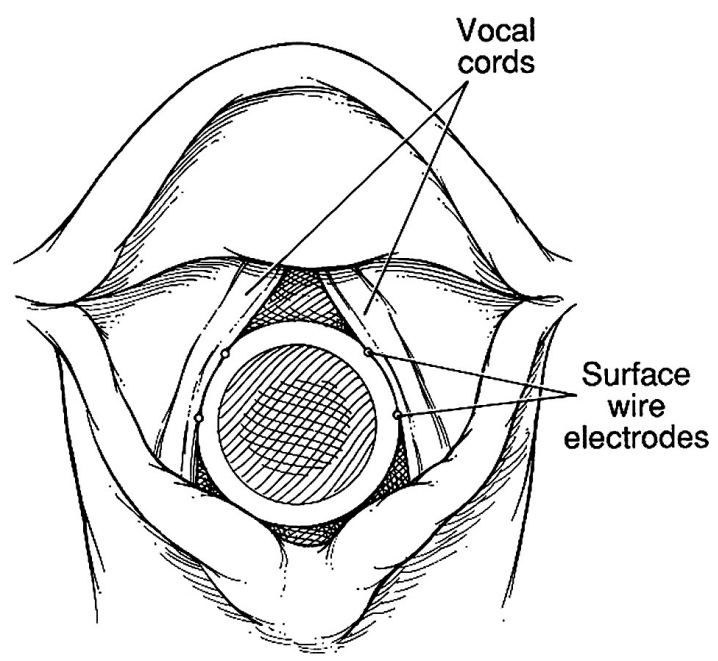

Figure 1. Recurrent laryngeal nerve monitoring

In this paper, 84 patients were given intubation through the visual laryngoscopes (Satum company, Canada). Visual laryngoscope can visually clearly exposed throat structure, reduce intubation injuries, improve the success of intubation time et al ${ }^{[5]}$ Due to the contact between the recording electrode and the vocal cords is directly related to the detection effect of recurrent laryngeal nerve.Visual laryngoscope intubation operations can help us get optimum depth of intubation and visually observe contact situation between the recording electrode and the vocal cords, so that we can adjust the tube in time. Lauren et al ${ }^{[4]}$ summarize the experience of apply Glidescope forNIM EMG endotracheal tube positioning for 3 cases of thyroid surgery, visual laryngoscopes provide good position for NIM EMG intubation, it is the ideal equipment in the operating room for the positing NIM EMG special endotracheal tubes, etc.

Dionigi et al [5] summarizes the 152 cases of thyroid surgery with NIM EMG intubation. There are 15 cases $(10 \%)$ were not satisfied with the EMG, it is necessary to further adjust the position of the tube, and there are $93 \%$ of 15 cases whose contact between the recorded vocal and electrodes is not available. The endotracheal tube malrotation accounted for $53 \%, 75 \%$ of those cases are clockwise rotation to the right. Also in our summary of the 84 patients, seven initial intubation patients $(8.3 \%)$ did not get a satisfactory EMG, 3 cases $(43 \%)$ of them as malrotation. SO intubation malrotation is the main cause of failed intubation. According to the experience of our department to rotate the tube counterclockwise $30^{\circ}$ can effectively prevent the malrotation. While to fix the endotracheal tube to the right side of the mouth is more convenient than the midline placement.

$\mathrm{Lu}^{[6]}$ has makes further analysis about the depth of NIM EMG intubation of 105 cases with thyroid surgery, THE analysis showed that for the male average depth of the tube was $20.6 \pm 0.97 \mathrm{~cm}$, and for the female the average depth of the tube was $19.6 \pm 1.0 \mathrm{~cm}$. With the increase in height we intubate deeper. The position of the head and neck changes often leads to the displacement of the endotracheal tube.Patients with head flexion may lead the tube deep into the bronchi, the ones whose head backward may make the tube fall into the pharynx, the it can lead maximum displacement to $1.9 \mathrm{~cm}$, and this is particularly evident for children, the rotation of the head can also cause the displacement of the tube tip to $0.7 \mathrm{~cm}{ }^{[7]}$. Thyroid surgery often require neck hyperextended, so it often leads electrode to move upward, resulting in intubation "inadequate."According to clinical observation, most cases will appear the situation of the recording electrodes move upward, so we often need to adjust the depth of intubation assisted by bronchoscopy Tsai et al gather 220 cases of thyroid surgery and divided them into two groups. The first group are the cases whose tube taped at the mouth after being intubated and then we make head move backward IN The second group,the tube is not fixed temporarily.After getting the head backward, adjusting and observing the position of the tube by endoscope then fix. At last two groups confirm endotracheal tube position by the endoscope.The ratio of Tube displacement is greater than $1 \mathrm{~cm}$ groups were $12.7 \%$ for the first group, 
the second group $3.6 \%{ }^{[8]}$. The way of intubation in second group is better than traditional way. It is recommended that before the induction of anesthesia patients should make the neck hyperextended position ,so this can avoid the displacement of head and neck posture, avoid to adjust the depth of the endotracheal tube frequently, achieve the best effect of nerve monitoring.

\section{Conclusion}

We got the satisfactory signals after adjusting the tube by using the visual laryngoscope.

\section{References}

1. Hui Sun,Xiaoli Liu,Yantao Fu. the application of Intraoperative nerve monitoring technology in the complex thyroid surgery [J]. Chinese Journal of Practical Surgery,2010,1;66-68(in Chinese)

2. Yi Wang,Yishen Zhao,Xiaoli Liu,Hui Sun. common fault and eliminating methods of laryngeal recurrent nerve in thyroid surgery monitoring system [C].2014 CTAThe third session of thyroid surgical peak BBS and the fifth international symposium on thyroid gland cavity mirror. (in Chinese)

3. Liangyu Wang,Digui Weng,Menghua Huang.GlidescopeVisual laryngoscope clinical analysis of 80 cases of emergency tracheal intubation [J]. Fujian Medical Journal,2016,38(1);12-14(in Chinese)

4. Lauren Berkow,Alan P.B.Dackiw,Ralph P.Tufano.Use of the GlideScopefor placement of arecurrent laryngeal nerve monitoring endotracheal tube[J].Journal of Clinical Anesthesia,2011,2;81-83

5. Dionigi G,Bacuzzi A,Boni L,et al. What is the learning curve for intraoperative neuromonitoring in thyroid surgery?[J]International Journal of Surgery,2008,6;7-12

6. I-Chen Lu,Koung-Shing Chu,Cheng-Jing Tsai,et al.Optimal depth of NIM EMG endotracheal tube for intraoperative neuro monitoring of the recurrent laryngeal nerve during thyroidectomy[J].World Journal of Surgery,2008,32;1935-1939

7. Tao Zhu,Yunxia Zuo,main translation. Anesthesiology. Version 5 [M].Beijing: People's Medical Publishing House, 2011;1679(in Chinese)

8. Cheng-Jing Tsai,Kuang-Yi Tseng,Fu-Yuan Wang,et al.Electromyographic endotracheal tube placement during thyroid surgery in neuromonitoring of recurrent laryngeal nerve[J].The Kaohsiung Journal of Medical Sciences,2011,27;96-101 